\title{
Characterization of Mouse Cell Line IMA 2.1 as a Potential Model System to Study Astrocyte Functions
}

Stefan Schildknecht ${ }^{1}$, Susanne Kirner ${ }^{1}$, Anja Henn ${ }^{1}$, Karlo Gasparic ${ }^{1}$, Regina Pape ${ }^{1}$, Liudmila Efremova ${ }^{1}$, Olaf Maier ${ }^{2}$, Roman Fischer ${ }^{2}$, and Marcel Leist ${ }^{1}$

${ }^{1}$ Doerenkamp-Zbinden Chair for in vitro Toxicology and Biomedicine, University of Konstanz, Konstanz, Germany;

${ }^{2}$ Institute for Cell Biology and Immunology, University of Stuttgart, Stuttgart, Germany

\begin{abstract}
Summary
Astrocytes are activated in most chronic neurodegenerative diseases associated with inflammatory events such as Parkinson's disease or Alzheimer's disease, but also in stroke. Due to an aging population worldwide, research efforts in these areas are likely to expand in the future. This will entail an increased demand for appropriate experimental models.

We introduce here the new immortalized mouse astrocyte cell line IMA 2.1 as an alternative to currently used primary astrocyte cultures. IMA 2.1 were directly compared with primary mouse astrocytes with respect to their response to proinflammatory stimuli, expression of typical astrocyte markers, and to the cell line's capacity to metabolize the parkinsonian toxin MPTP to its toxic metabolite MPP ${ }^{+}$. Under inflammatory conditions, mimicked with the addition of a cytokine mix, IMA 2.1 responded similarly to primary astrocytes with $m R N A$ upregulation, expression of iNOS and COX-2, and the release of various inflammatory mediators. Analysis of astrocytic markers indicated that IMA 2.1 represent a relatively early, GFAP-negative stage of astrocyte development. Moreover, conversion of MPTP by monoamine oxidase-B proceeded in IMA at least as quickly as in primary cells. For all endpoints investigated, the cell line IMA 2.1, derived from a single clone, delivered reproducible results over a period of several years and allowed upscaling of experiments due to its easy handling compared with primary cells.
\end{abstract}

Keywords: astrocytes, LUHMES, inflammation, Parkinson's disease, MPTP

\section{Introduction}

Astrocytes are highly differentiated cells of the central nervous system that serve numerous functions, such as nutrient supply of neurons, regulation of cerebral blood flow, orchestration of neuronal growth and differentiation, maintenance of extracellular glutamate levels, and ion and liquid balance (Kettenmann and Ransom, 2005). They are furthermore involved in inflammatory processes in the central nervous system that are observed not only following the invasion of pathogens but also in association with chronic neurodegenerative diseases such as Alzheimer's disease (AD) or Parkinson's disease (PD) (Falsig et al., 2008). The primary effector cells of the brain in response to bacteria are microglia (Lund et al., 2005, 2006). They release proinflammatory cytokines that trigger the subsequent activation of astrocytes as a second line of defense (Lee et al., 1993; Henn et al., 2011; Falsig et al., 2006). Following stimulation, both cell types become rich sources of cytokines and prostanoids (Murphy et al., 1988; Meeuwsen et al., 2003). Moreover, they produce the free radicals nitric oxide $\left({ }^{\bullet} \mathrm{NO}\right)$ and superoxide $\left({ }^{\bullet} \mathrm{O}_{2}^{-}\right)$, which can lead finally to the formation of several cytotoxic species such as peroxynitrite $\left(\mathrm{ONOO}^{-}\right)$or hydroxyl radicals $\left({ }^{\bullet} \mathrm{OH}\right)$ (Brown and Neher, 2010; Beckman and Koppenol, 1996). These molecules can attack and modify cellular proteins, lipids, and DNA in neighboring neurons, particularly in association with chronic brain diseases, as illustrated in numerous reports (Schildknecht et al., 2011).

In a widely used in vivo model of $\mathrm{PD}$, astrocytes are responsible for the conversion of the parkinsonian toxin 1-methyl-4phenyl-1,2,3,6-tetrahydropyridine (MPTP) into its active metabolite 1-methyl-4-phenylpyridinium $\left(\mathrm{MPP}^{+}\right)$(Ransom et al., 1987; Di Monte et al., 1991). The responsible enzyme has been identified as monoamine oxidase-B (MAO-B) (Langston et al., 1984). After release from astrocytes, $\mathrm{MPP}^{+}$is selectively taken up by dopaminergic neurons leading to their death (Pöltl et al., 2011; Schildknecht et al., 2009). The resultant symptoms and pathology recapitulate key features observed in PD patients. The active toxin $\mathrm{MPP}^{+}$is widely used in cellular cultures of neuronal cells. However, the presence of glial cells as such can have a profound impact on the degeneration process of neurons

Received February 14, 2012; accepted in revised form March 28, 2012 
exposed to $\mathrm{MPP}^{+}$or other toxins. To model the in vivo situation more faithfully, co-culture models composed of astrocytes and neurons are desirable. They would allow studies of the process of MPTP conversion by astrocytes, as well as the subsequent uptake and degeneration of neurons in a defined and easily accessible in vitro model. Primary astrocytes are used in most currently established co-culture systems (Roqué et al., 2011). Availability and use of a defined and well-characterized astrocyte cell line that can be applied in such experimental models would avoid the disadvantages of primary cultures.

Primary rodent astrocytes have been used for many in vitro disease models and to obtain biochemical insight into astrocyte function (Kim and Magrané, 2011; Falsig et al., 2004b; Christiansen et al., 2011). Although highly sophisticated isolation procedures have been developed, batch-to-batch variations and contamination with microglia represent considerable shortcomings in the use of primary cultures (Hansson, 1986). As primary astrocytes hardly proliferate, the high demand for animals puts an additional burden on this type of cellular model. For example, highly purified primary astrocyte preparations, as used for gene expression profiling (Falsig et al., 2004a, 2006) may yield less than 1 million cells per mouse and a single study may require the sacrifice of several hundreds of mice. In order to find alternative approaches, numerous attempts have been undertaken to generate astrocyte cell lines by retroviral transduction of immortalizing oncogenes. The first approach dates back to 1990, when progenitor cells isolated from mouse brains were transduced with the myc-oncogene that allowed the generation of cell lines with neuronal and glial phenotypes (Ryder et al., 1990). In a more sophisticated approach, mouse cortical astrocytes of varying differentiation stages were immortalized by SV40 T antigen, and this allowed the generation of mature and immature mouse astrocyte lines (Frisa et al., 1994). In an attempt to generate an immortalized human astrocyte cell line, astrocytes obtained from human fetal brain have been purified and transfected with the SV40 T antigen. However, it was noted that the high proliferation capacity of this newly generated A735 line was associated with downregulation of GFAP expression and subsequent loss of other astrocyte specific markers (Price et al., 1999) and thus that cell line does not represent an adequate substitute for primary cells. In a new attempt to provide an alternative to primary astrocyte cultures, we immortalized cortical astrocytes of wildtype BALB/c mice with the SV40 large T antigen, created single cell clones, and selected the clone IMA 2.1 based on its ability to respond to proinflammatory cytokines and its expression of functional monoamine oxidase-B (MAO-B).

During more than three years of use of IMA 2.1 in our laboratory, its phenotype and response pattern towards cytokines remained stable. This makes the IMA 2.1 a reliable new model for certain astrocyte studies (Leist et al., 2010). In the present manuscript, we provide data on the characterization of this new cell line and suggest the IMA 2.1 cell line as a useful tool for a variety of in vitro studies of astrocyte functions including astrocyte-neuron crosstalk. It is intended as an overview for interested researchers to enable a first judgment on whether IMA 2.1 may be useful for their planned investigations.

\section{Animals, materials, and methods}

\subsection{Immortalization of primary astrocytes from BALB/c mice}

Cerebral cortices were isolated from one- or two-day-old mice. After removal of the meninges, cortices were chopped into small pieces with a razor blade and further homogenized by enzymatic digestion in PBS/0.5\% trypsin/EDTA (Gibco/Invitrogen, Karlsruhe, Germany), 0.5\% DNase I (Sigma-Aldrich, Steinheim, Germany) for $15 \mathrm{~min}$ at $37^{\circ} \mathrm{C}$. Trypsin was inactivated by addition of fetal calf serum (FCS; PAN Biotech, Aidenbach, Germany) and tissue was triturated using a Pasteur pipette. The homogenates were seeded on poly-D-lysine hydrobromide (PDL; $10 \mu \mathrm{g} / \mathrm{ml}$; Sigma-Aldrich, MW 30-70 kDA) coated culture flasks at a density of approximately 40,000 cells $/ \mathrm{cm}^{2}$ in DMEM (Gibco/Invitrogen) supplemented with 10\% FCS, 2 mM L-Glutamin (Invitrogen) and 1\% penicillin/ streptomycin (Invitrogen). After 8 days in culture, an astrocyte layer, overgrown by numerous microglial cells, oligodendrocyte precursor cells, and oligodendrocytes can be observed. To prepare purified astrocyte cultures, oligodendrocytes and surface microglia were flushed away from the astrocyte layer. The remaining microglia were removed by trypsinization of the primary cultures and differential adhesion for $30 \mathrm{~min}$ on uncoated culture dishes. The astrocytes remaining in the supernatant were collected, and approximately $0.5 \times 10^{6}$ cells were plated in PDL-coated 6-well plates. Cells were grown to confluency in culture medium without antibiotics. On the day of transfection, medium was changed to Opti-MEM I (Gibco). Lipofectamine 2000 (Invitrogen) was diluted 1:50 in Opti-MEM I and incubated for $5 \mathrm{~min}$ at room temperature. The plasmid psV3neo (Chang et al., 1986), coding for the large $\mathrm{T}$ antigen from SV40 (500 ng) was diluted in Opti-MEM I and mixed in a ratio of 1:1 with the lipofectamine dilution. The resulting transfection complexes were preincubated at room temperature for an additional $20 \mathrm{~min}$, the transfection mixture (500 $\mu 1 /$ well) was added carefully to the cells and then incubated at $37^{\circ} \mathrm{C}$. After $6 \mathrm{~h}$, the transfection mixture was replaced by standard cell culture medium and the cells were incubated for an additional $24 \mathrm{~h}$. Stably transfected cells were selected with $800 \mu \mathrm{g} / \mathrm{ml}$ geneticin (G418; Invitrogen). The resultant immortalized mouse astrocytes (IMA) were cultured at $37^{\circ} \mathrm{C}$ $\left(5 \% \mathrm{CO}_{2}\right)$ in DMEM Glutamax (high glucose) (GIBCO) with $5 \%$ FCS (fetal calf serum, from PAA) without antibiotics. They were trypsinized for 2 min with $0.5 \%$ trypsin/DMEM every 2-3 days and reseeded at a ratio of 1:5 or 1:10. Experiments were performed in DMEM containing $2 \%$ FCS.

\subsection{Primary astrocyte cultures}

Primary cortical astrocytes were generated from BALB/c mice bred at the animal facility of the University of Konstanz, Germany. All mice were housed at $22^{\circ} \mathrm{C}$ and $55 \%$ relative humidity in a $12 \mathrm{~h}$ day/night rhythm with free access to food and water according to national regulations and EU Directive 2010/63/EU. Primary cortical astrocytes were prepared from mouse pups at 24-48 $\mathrm{h}$ post partum, as described earlier (Henn et al., 2011). In brief, brains were removed, the cortices were dissected out, and 
hippocampi and meninges were carefully removed before digestion with trypsin and DNase. After trituration, the cell suspension was carefully layered over a $30 \%$ Percoll solution and centrifuged at $150 \mathrm{x} \mathrm{g}$ for $10 \mathrm{~min}$. The astrocyte fraction was recovered, washed, and resuspended in DMEM (high glucose), $20 \% \mathrm{FCS}, 100 \mathrm{U} / \mathrm{ml}$ penicillin and $100 \mu \mathrm{g} / \mathrm{ml}$ streptomycin. Cells were seeded at a density of 2 million cells (corresponding to 4 brains) per T175 flask, and cultured at $37^{\circ} \mathrm{C}$ in a $5 \% \mathrm{CO}_{2}$ atmosphere. The medium was changed after 4 days, and subsequently every second day. After 14 days in primary culture, cells were trypsinized and incubated in DMEM for $45 \mathrm{~min}$ at room temperature in a T75 flask for differential adhesion of any residual microglia. Non-adherent cells (astrocytes) were reseeded in DMEM plus 10\% FCS, containing $4.5 \mathrm{~g} / \mathrm{l}$ D-glucose and $2 \mathrm{mM}$ Glutamax, and cultured in multiwell dishes for 7-9 days before use in experiments. This protocol was shown to result in microglia or oligodendrocyte contaminations below our detection limit $(<1 \%)$ by FACS, PCR, or immunostaining.

\subsection{Co-culture model}

Primary astrocytes or IMA were seeded into 24-well plates (Nunclon, Nunc, Roskilde, Denmark), and when confluency was reached, the cells were maintained for 7 additional days. Then, predifferentiated (2-days) human mesencephalic cells (LUHMES) were seeded on top of the glial cell layer at a density of $180,000 / \mathrm{cm}^{2}$ in Advanced DMEM (Gibco), containing $2 \mathrm{mM}$ L-glutamine, $1 \times \mathrm{N} 2$ supplement, and $1 \mu \mathrm{g} / \mathrm{ml}$ tetracycline for 4 more days. LUHMES cells were previously characterized with respect to their applicability as an in vitro model of terminally differentiated dopaminergic neurons (Schildknecht et al., 2009; Scholz et al., 2011; Lotharius et al., 2005). To selectively visualize the neurons in these co-cultures, cells were fixed with $4 \%$ paraformaldehyde (PFA) for $15 \mathrm{~min}$ and permeabilized with $0.2 \%$ Triton X-100 in PBS for 10 min. Following blocking with $1 \%$ bovine serum albumin (BSA) in PBS for $1 \mathrm{~h}$, LUHMES were stained with an antibody against the neuronal class of $\beta$-III-tubulin (TUJ-1; Convance, mouse, 1:1000) and fluorescence-labeled secondary antibodies.

\subsection{Translocation of NF- $\mathrm{KB}$}

Cells were plated in 96-well plates at 15,000 cells/well and cultured in DMEM plus 2\% FCS. The cells were stimulated as indicated, then fixed with $4 \%$ PFA for $15 \mathrm{~min}$, permeabilized with $0.1 \%$ Triton $\mathrm{X}-100$ for 10 min, blocked with $10 \%$ FCS for $1 \mathrm{~h}$, immunostained with purified monoclonal mouse

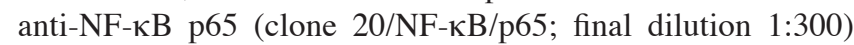
antibody (BD Transduction Laboratories) over night, and detected with anti-mouse IgG Alexa-488 secondary antibody. Images were recorded with a high-resolution CCD camera on a Cellomics ArrayScan ${ }^{\mathrm{TM}}$, which is based on an automated Zeiss Axiovert-100 microscope equipped with a $20 \mathrm{x}$ objective and a FITC/H-33342 filter set $\left(\lambda_{\text {ex-1 }}=365 \mathrm{~nm}, \lambda_{\text {ex }-2}=475 \mathrm{~nm}\right.$, $\left.\lambda_{\mathrm{em}}=535 \mathrm{~nm}\right)$. Nuclei, stained with H-33342, were imaged first (channel 1) for automated focusing and identification of valid objects. Subsequently, the stained antigens were imaged in the corresponding fields (channel 2). Based on these data sets, the nuclear translocation of NF- $\mathrm{KB}$ was quantified for each cell with the predefined algorithm "nuclear translocation," essentially as described earlier (Henn et al., 2009, 2011).

\subsection{Flow cytometry}

Cells from 6-well plates were trypsinized and washed twice with PBS, containing 10\% FCS. They were centrifuged for $5 \mathrm{~min}$ at $500 \mathrm{x} \mathrm{g}$. FITC-conjugated anti-TLR2 antibody (clone 6C2; eBioscience; Alasdair Stewart, U.K) diluted in $2 \%$ FCS/PBS to a concentration of $1 \mu \mathrm{g}$ per million cells (in $200 \mu \mathrm{l}$ ) was added for $50 \mathrm{~min}$ at $4^{\circ} \mathrm{C}$. An appropriate isotype control was used in a similar way. Cells were washed twice and fixed with $0.5 \%$ PFA. For each sample, a minimum of 10,000 cells within the gated cell population was analyzed using a flow cytometer (Accuri Cytometers, Cambs, U.K.) and the corresponding software CFlow. The percentage of positively stained cells relative to isotype control (maximum $1 \%$ positive staining) was determined.

\subsection{RNA extraction, RT-PCR, and quantitative PCR}

Total RNA was extracted with TRIzol from Invitrogen (Darmstadt, Germany) and $1 \mu \mathrm{g}$ of total RNA was reverse transcribed with Superscript $^{\mathrm{TM}}$ II Reverse Transcriptase (Invitrogen, Darmstadt, Germany) using random hexamers and Oligo-dT-Primer in a $20 \mu \mathrm{l}$ reaction according to the manufacturer's protocol. PCR amplification of the cDNA was quantified using the Platinum ${ }^{\circledR}$ SYBR $^{\circledR}$ Green qPCR SuperMix-UDG kit (Invitrogen, Darmstadt, Germany). The threshold cycle $\left(\mathrm{C}_{\mathrm{T}}\right)$ was determined for each sample using the iCycler MyiQ qPCR detection system (BioRad Laboratories, Hercules, CA, USA) and corresponding data analysis software. The housekeeping gene control was gapdh. The relative cDNA levels were compared among different groups using the delta-delta $\mathrm{C}_{\mathrm{T}}$ method. Absolute levels were calculated by the delta $\mathrm{C}_{\mathrm{T}}$ method. The following primer sequences were used in the study:

Aqp 4: sense: $5^{\prime}$-GCT CAG AAA ACC CCT TAC CTG TGG3', antisense: 5'-TTC CAT GAA CCG TGG TGA CTC C-3'; Cnx 43: sense: $5^{\prime}$-CCA CTC TCA CCT ATG TCT CCT CC3', antisense: 5'-CGT CAG GGA AAT CAA ACG GCT GG3'; COX-2: sense: 5'-CCA TTG AAC CTG GAC TGC AGA AGG C-3', antisense: $5^{\prime}$-CGG AAC TAA GAG GAG CAG CAA TGC-3'; GAPDH: sense 5'-CAG GCA GCT CAG GTT GAC TCT G-3', antisense 5'-GGA TGC AGG GAT GAT GTT C-3'; GFAP: sense 5'-CGA GAT CGC CAC CTA CAG GAA GC-3', antisense: $5^{\prime}$-CCT TAA TGA CCT CTC CAT CCC GC-3'; GLAST: sense 5'-CTC TAC GAG GCT TTG GCT GC3', antisense: 5'-GAG GCG GTC CAG AAA CCA GTC-3'; GLT-1: sense: 5'-GCA GCC ATC TTC ATA GCC CAAATG-3', antisense: $5^{\prime}$-CTC ATT CTA TCC AGC AGC CAG TCC-3'3 GS: sense: 5'-GTG TGT GGA AGA GTT ACC TGA GTG G-3', an-

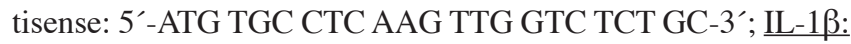
sense: 5'-TTT TTG TTG TTC ATC TCG GAG CCT GTA G-3', antisense: 5'-GAG CAC CTT CTT TTC CTT CAT CTT TG3'; IL-6: sense: 5'-CCT CTG GTC TTC TGG AGT ACC ATA GC-3', antisense: 5'-GGA GAG CAT TGG AAA TTG GGG TAG G-3’; IL-23: sense: 5'-CAT GCA CCA GCG GGA CAT ATG-3', antisense: 5'-CAG ACC TTG GCG GAT CCT TTG- 
3'; Kir4.1: sense: 5'-GTA GAC ACA GCC TCA GAC AGC C-3', antisense: 5'-GGT AGG AAG TGC GAA CTT GGC-3'; MAO-B: sense: 5'-AAC AGT ATT CTG GGG GCT GCT ACA3', antisense: 5'-AGG GAC ATC CAA AGA TTC TGG TTC TG-3'; 1 MCP-1: sense: 5'-CAT AGC AGA ACA GAA GAA CAG-3', antisense: 5'-GTC TTC GGA GTT TGG GTT TG-3'; Nestin: sense: 5'-CTG GAA GGT GGG CAG CAA CT-3', antisense: 5'-ATT AGG CAA GGG GGA AGA GAA GGT G-3'; NOD-2: sense: 5'-CAG GCA GCT CAG GTT GAC TCT G-3', antisense: 5'-TAG AAA GCG GCA AAA AAG CAC TGG AAG-3'; NOS-2: sense: 5'-TTG CCA CGG ACG AGA CGG ATA GG-3', antisense: 5'-GGG GTT GTT GCT GAA CTT CCA GTC-3’; S100ß: sense: 5'-GGT TGC CCT CAT TGA TGT CTT CCA C-3', antisense: 5' -CTT CCT GCT CCT TGA TTT CCT CCA G-3'; TLR-2: sense: $5^{\prime}$-CCT CCG TCT TGG AAT GTC ACC AGG-3', antisense: 5'-GAG CCA CGC CCA CAT CAT TC-3'; TLR-3: sense: 5'-GCC CCC TTT GAA CTC CTC TTC-3', antisense: 5'-AGA TCC TCC AGC CCT CGA TG-3'; TNF- $\alpha$ : sense: $5^{\prime}$-TAG CAA ACC ACC AAG TGG AGG AG3', antisense: 5'-GCA GCC TTG TCC CTT GAA GAG AA-3'; TYKi: sense: 5'- GGA TGC GCT GCA CAC CAA TT-3', antisense: 5'-TAG CTC CTT GGC CTC CGT TTG-3'.

\subsection{Measurements of prostanoids and nitrite in cell culture supernatants}

Prostaglandin $\mathrm{E}_{2}\left(\mathrm{PGE}_{2}\right)$ was determined by using commercially available EIA-kits (Assay Designs, MI, USA) according to the manufacturer's instructions. Nitrite $\left(\mathrm{NO}_{2}^{-}\right)$, the stable autoxidation product of ${ }^{\bullet} \mathrm{NO}$, was measured by the Griess assay. Briefly, $30 \mu 112.5 \mu \mathrm{M}$ sulfanilamide (Sigma) and $30 \mu 16 \mathrm{M} \mathrm{HCl}$ were mixed with $200 \mu \mathrm{l}$ cell culture supernatant at room temperature and incubated for $5 \mathrm{~min}$. Absorbance was measured before and after the addition of $25 \mu \mathrm{N}$-(1-naphthyl)ethylenediamide $(12.5 \mu \mathrm{M})($ Sigma) at $560 \mathrm{~nm}$ using a microtiter plate reader. Nitrite concentrations were calculated from a $\mathrm{NaNO}_{2}$ standard curve in the range of $0.5-10 \mu \mathrm{M}$.

\subsection{HPLC analysis}

Detection of MPTP, MPP ${ }^{+}$, and $\mathrm{MPDP}^{+}$was performed on a Kontron system (Goebel Analytic, Au/Hallertau, Germany) composed of a model 520 pump, model 560 autosampler, models 535 and 430 diode array detectors, set at $245 \mathrm{~nm}$ for MPTP, $295 \mathrm{~nm}$ for $\mathrm{MPP}^{+}$, and $345 \mathrm{~nm}$ for $\mathrm{MPDP}^{+}$.

Samples were acidified with $9 \mu \mathrm{l}$ perchloric acid (70\%) per ml volume of sample and centrifuged at $10,000 \mathrm{x} g$ for $15 \mathrm{~min}$. The supernatant was filtered through a Chromaphil PET-20/15MSfilter with $0.2 \mu \mathrm{m}$ pore size from Macherey Nagel (Düren, Germany).

Separation was carried out on a $\mathrm{C} 18$ nucleosil column (250 x $4.6 \mathrm{~mm} ; 5 \mu \mathrm{m}$ particle size) from Macherey Nagel (Düren, Germany) at room temperature. The mobile phase consisted of acetonitrile : distilled water : triethylamine : sulfuric acid (12.50: $86.18: 1.04: 0.28, \mathrm{v} / \mathrm{v}, \mathrm{pH} 2.3)$. The mobile phase was degassed with an online vacuum degasser and delivered isocratically at a flow rate of $1 \mathrm{ml} / \mathrm{min}$ at an average pressure of 145 bar. Data analysis was performed with Geminyx II software (Goebel Analytic).

\subsection{Monoamine oxidase (MAO) activity assay}

The assay is based on the oxidation of kynuramine to 4-hydroxyquinoline by MAO. Cells were homogenized in PBS, and total protein content was adjusted to $1 \mathrm{mg} / \mathrm{ml}$. Then, $180 \mu 1$ of cell homogenate were supplemented with $20 \mu 1$ substrate kynuramine (Sigma) in PBS to yield a final concentration of $100 \mu \mathrm{M}$. In parallel, a standard curve of 4-hydroxyquinoline $(0-100 \mu \mathrm{M})$ was prepared. After $2 \mathrm{~h}$ of incubation, the reaction was terminated by the addition of $80 \mu 1$ perchloric acid $(400 \mathrm{mM})$. Samples were centrifuged at $12,000 \mathrm{x}$ g for $1 \mathrm{~min}, 200 \mu 1$ supernatant was transferred into a new tube and mixed with $400 \mu 1 \mathrm{NaOH}$ (1 M). Samples of the standard curve were treated accordingly and fluorescence was detected at $\lambda_{\mathrm{ex}} 315 \mathrm{~nm} / \lambda_{\mathrm{em}} 380 \mathrm{~nm}$.

\subsection{Cyclooxygenase (COX) activity}

$\mathrm{COX}$ activity was determined by the conversion of the ${ }^{14} \mathrm{C}$ labeled substrate arachidonic acid $\left({ }^{14} \mathrm{C}-\mathrm{AA}\right)$ to prostanoids (Schildknecht et al., 2008). Cells were washed twice, collected in cold PBS and centrifuged at $1000 \mathrm{x} \mathrm{g}$ for $3 \mathrm{~min}$. The pellet was dissolved in lysis buffer (20 mM HEPES, $1 \%$ Triton X-100, $1 \%$ aprotinin, $10 \%$ glycerol, $\mathrm{pH} 7.5$ ) for $30 \mathrm{~min}$ on ice. Following centrifugation at $12,000 \mathrm{x} \mathrm{g}$ for $1 \mathrm{~min}$, the supernatant was incubated with reaction buffer $(80 \mathrm{mM}$ Tris-HCl, 0.1 $\mathrm{mM}$ phenol, $5 \mu \mathrm{g} / \mathrm{ml}$ hematin, $5 \mu \mathrm{M}{ }^{14} \mathrm{C}-\mathrm{AA}, \mathrm{pH} 8.0$ ) for 20 $\mathrm{min}$. The reaction was terminated by the addition of ethyl acetate/ $2 \mathrm{M}$ citric acid (30:1). After vortexing for at least $1 \mathrm{~min}$, the organic phase was collected, evaporated and used for analysis on silica TLC plates (Silica 60, Merck, Darmstadt, Germany) by chromatography. The mobile phase consisted of ethyl acetate : 2,2,4-trimethylpentane : acetic acid : water (110:50:20:100). Plates were dried and used to expose a PhosphorImager ${ }^{\mathrm{TM}}$ screen overnight. For reading the screen, a PhosphorImager ${ }^{\mathrm{TM}}$ system from Molecular Dynamics, USA was used. Quantification was performed by the detection of total prostanoid formation utilizing ImageQuant ${ }^{\mathrm{TM}}$ software.

\subsection{Western blot analysis}

Cells were lysed in RIPA buffer (50 mM Tris-base, $150 \mathrm{mM}$ $\mathrm{NaCl}, 1 \mathrm{mM}$ EDTA, $0.25 \%$ sodium deoxycholate, $1 \%$ NP40, $1 \mathrm{mM} \mathrm{Na} 3 \mathrm{VO}_{4}, 50 \mathrm{mM} \mathrm{NaF}, \mathrm{pH}$ 7.5). Protein determination was performed by using a BCA protein assay kit (Pierce). After loading of $25 \mu \mathrm{g}$ protein to a $8 \%$ polyacrylamide SDS gel, proteins were transferred onto nitrocellulose membranes (Amersham, Buckinghamshire, UK). Loading and transfer were controlled by brief Ponceau staining. Washed membranes were then blocked with $5 \%$ milk in PBS-Tween $(0.1 \%)$ for $2 \mathrm{~h}$. Primary antibodies were added at $4^{\circ} \mathrm{C}$ over night (COX-2: Cayman, polyclonal, 1:1000; NOS-2: BD Transduction, polyclonal rabbit 1:5000; $\beta$-actin: Sigma, monoclonal mouse 1:2000). Following washing steps with PBS-Tween, horseradish peroxidaseconjugated secondary antibodies were added for $1 \mathrm{~h}$ at room temperature. For visualization, ECL western blotting substrate (Pierce) was used. As secondary antibodies, anti-mouse HRP 1:5000, Jackson Immuno Research and anti-rabbit HRP 1:5000, GE Healthcare were used. 


\subsection{Inflammatory stimulation}

IMA or primary astrocytes were stimulated with cytokines or the TLR-2 ligand FSL-1 (Invitrogen, San Diego, CA). Murine TNF- $\alpha$, IL- $1 \beta$, and IFN- $\gamma$ were obtained from R\&D Systems (Wiesbaden, Germany). The combination of IL-1 $\beta$ (10 ng/ml), TNF- $\alpha(10 \mathrm{ng} / \mathrm{ml})$, and IFN- $\gamma(20 \mathrm{ng} / \mathrm{ml})$ was termed complete cytokine mix (CCM).

\subsection{Immunocytochemistry}

Cells were grown on $10 \mathrm{~mm}$ glass cover slips (Menzel, Braunschweig, Germany) in 24-well plastic cell culture plates (Nunclon $\left.^{\mathrm{TM}}\right)$. Following treatment, cells were fixed with 4\% PFA for 20 min at $37^{\circ} \mathrm{C}$ and washed with PBS. After blocking with $1 \%$ BSA (Calbiochem, San Diego, CA) for $1 \mathrm{~h}$, primary antibodies (anti-CD44, 1:500, rat monoclonal, Caltag Laboratories; antinestin, 1:500, rat monoclonal, Millipore) were added in PBSTween $(0.1 \%)$ at $4{ }^{\circ} \mathrm{C}$ over night. Secondary antibodies (anti-rat IgG-Alexa 488, Invitrogen, Darmstadt, Germany) were added for 45 min at RT. For visualization, an Olympus IX 81 microscope (Hamburg, Germany) equipped with an F-view CCD camera was used. Nuclei were stained with H-33342 $(1 \mu \mathrm{g} / \mathrm{ml})$ for $15 \mathrm{~min}$ prior to the final washing step. For image processing, Cell P software (Olympus) was used.

\subsection{Statistics}

NF-кB translocation data are based on at least 200 valid cells per well and are indicated as the means \pm SEM of at least three wells from independent experiments. Flow-cytometry analysis was done in duplicates. Nitrite and $\mathrm{PGE}_{2}$ detection, enzyme activity assays and mRNA experiments were all performed in triplicates using independent cell preparations. Statistical analysis was performed with GraphPad Prism software (Version 4.03), using t-test or one-way ANOVA with Bonferroni's post-hoc test, as appropriate.

\section{Results}

\subsection{Characterization of inflammatory responses}

Initially, we used a pool of IMA, obtained by selection of the transfected cells by antibiotics, and then expanded the cells by passaging in normal culture medium. After stable growth conditions were reached, such cells were tested for their capacity to react to inflammatory cytokines. Altogether, six cell pools from different transfections were used (IMA 1-6) to assess $\mathrm{NF}-\kappa \mathrm{B}$ translocation from the cytosol to the nucleus. This signaling response is usually observed after stimulation of astrocytes with various cytokines as well as toll-like receptor (TLR) ligands. Here, it was quantitatively assessed $30 \mathrm{~min}$ after stimulation with IL- $1 \beta$, TNF- $\alpha$, IFN- $\gamma$, or a combination of all three cytokines (complete cytokine $\operatorname{mix}=\mathrm{CCM}$ ). TNF- $\alpha$ or IL$1 \beta$ stimulation alone was sufficient to evoke maximal NF- $\kappa \mathrm{B}$ translocation (Fig. 1A). In these experiments, IMA 2 showed the best responses, and they were used thereafter. In order to obtain a homogeneous cell population, IMA 2 were diluted to the single cell level and different clones originating from this procedure were generated and compared. Five IMA 2 sub-

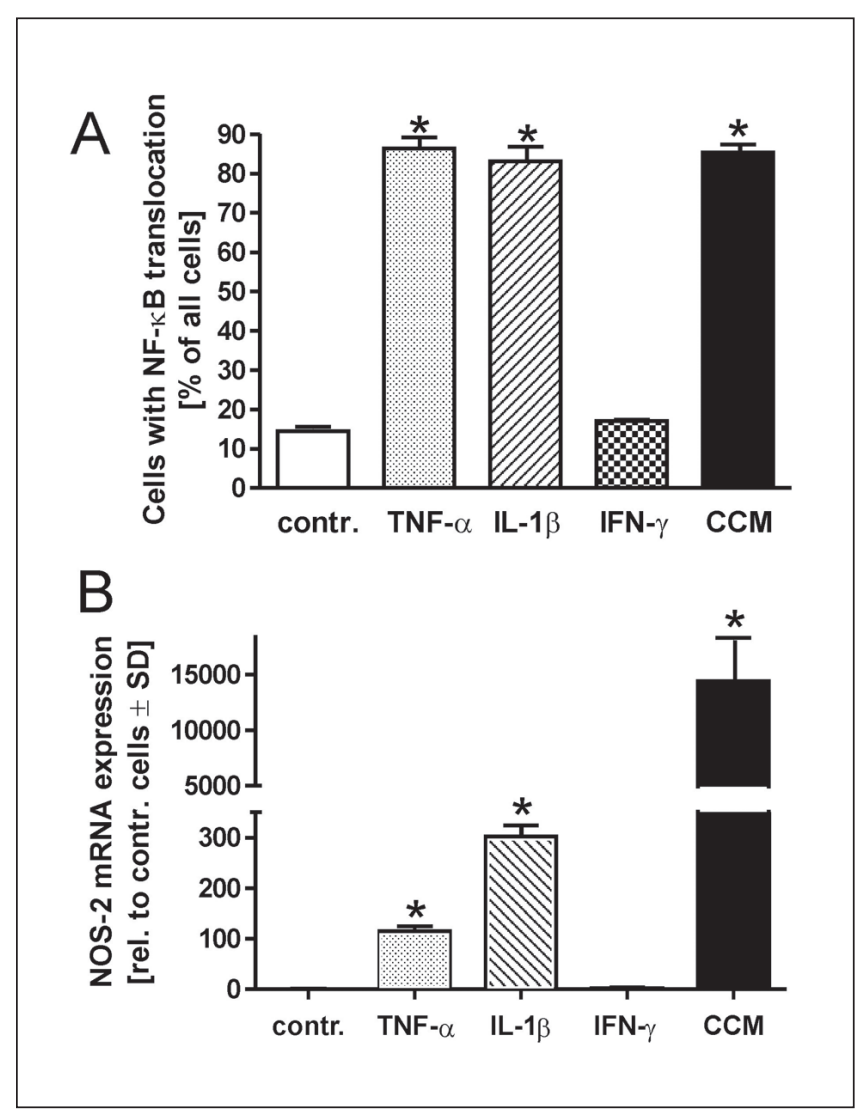

Fig. 1: Inflammatory response to different cytokines (A) IMA 2.1 were stimulated with IL-1 $\beta(10 \mathrm{ng} / \mathrm{ml})$, TNF-a $(10 \mathrm{ng} /$ $\mathrm{ml})$, or IFN-ץ $(20 \mathrm{ng} / \mathrm{ml})$, or by a complete cytokine mix (CCM) composed of all three cytokines for $30 \mathrm{~min}$. Cells were then fixed and stained with an anti-NF- $x B$ p65 antibody. Quantitative assessment of NF-KB translocation from the cytosol to the nucleus was performed with an automated microscope (Cellomics Array Scan). This method allows detection of NF-KB translocation on the single cell level. Data are displayed as percentage of cells positive for NF-KB translocation compared to all cells detected. (B) IMA 2.1 were stimulated with cytokines as indicated for $16 \mathrm{~h}$, nitric oxide synthase-2 (NOS-2) mRNA was detected as representative for an early immediate gene. Data are expressed as means $\pm S D$ of quadruplicate samples. Statistical significance was determined by one-way ANOVA followed by Bonferroni's post-hoc test. ${ }^{*} P<0.05$

clones (designated IMA 2.1 - IMA 2.5) were investigated with respect to their response to different cytokines. In all subclones, IL-1 $\beta$, TNF- $\alpha$, or CCM were observed to trigger strong NF- $x \mathrm{~B}$ translocation to the nucleus. Clone 2.1 showed slightly higher growth rates and therefore was selected for all further experiments and characterizations.

The inducible isoform of nitric oxide synthase (NOS-2) was chosen as a representative example for pathophysiologically-relevant astrocyte enzymes. NOS-2 mRNA expression was slightly increased after $16 \mathrm{~h}$ of stimulation by TNF- $\alpha$ or IL-1 $\beta$ (Fig. 1B). Combination of the two cytokines did not further increase NOS-2 mRNA, and IFN- $\gamma$ had no effect, when 


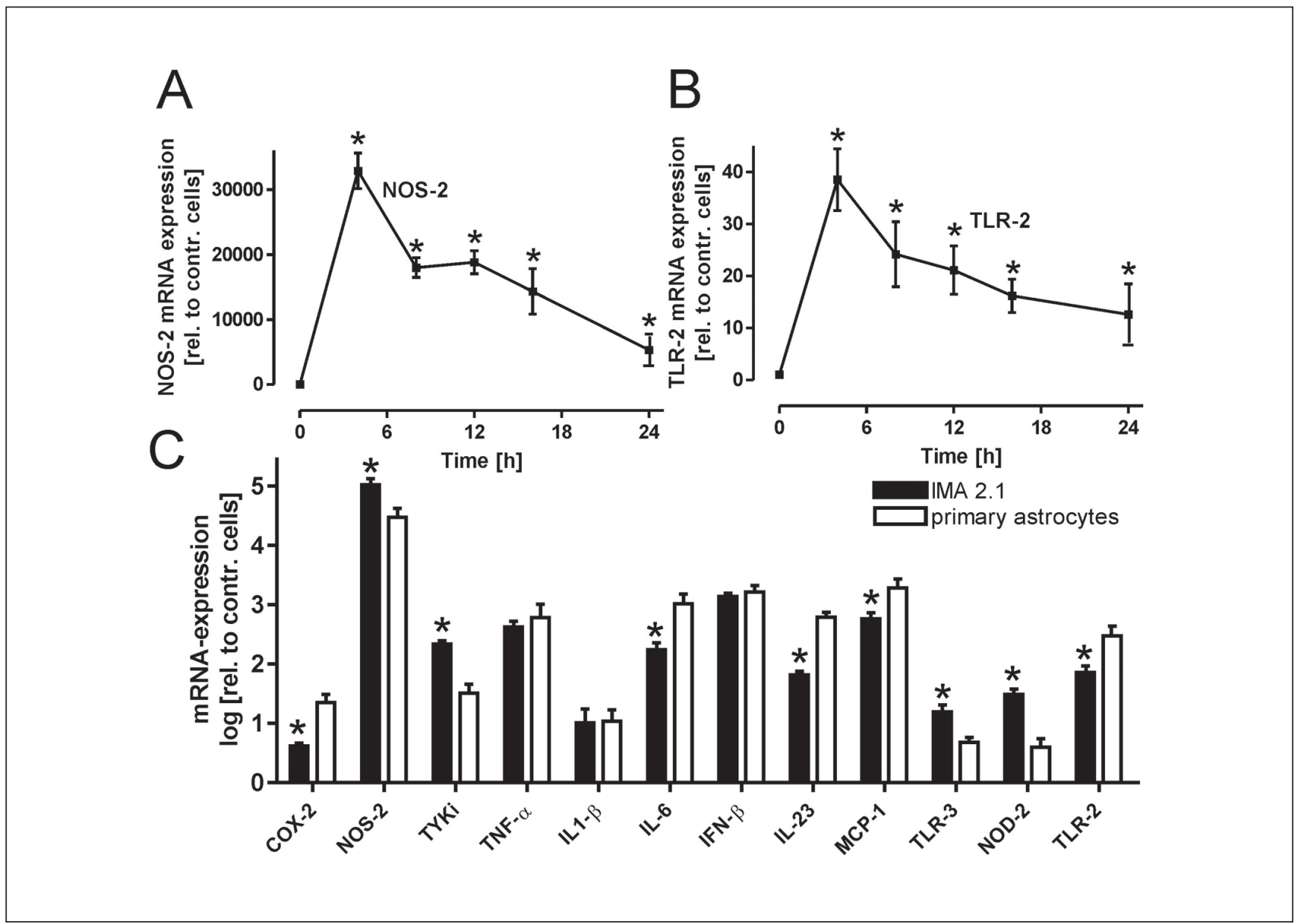

Fig. 2: Time course and spectrum of inflammatory responses

IMA 2.1 were stimulated by CCM for the time intervals indicated. Total mRNA was isolated and served as template for cDNA synthesis and PCR amplification. (A+B) The inducible proinflammatory enzyme nitric oxide synthase-2 (NOS-2) as well as toll-like receptor 2 (TLR-2) were determined by real-time PCR. Data are normalized to GAPDH expression. (C) Cells were activated by CCM for $4 \mathrm{~h}$, data were normalized to GAPDH and displayed as $\triangle \Delta$ ct values relative to the respective mRNA expression in unstimulated cells. COX-2 = cyclooxygenase-2; NOS-2 = nitric oxide synthase-2; TYKi = inducible thymidylate kinase; TNF- $\alpha=$ tumor necrosis factor $\alpha$; IL-1 $\beta$ = interleukin-1 $\beta$; IL-6 = interleukin 6; IFN- $\beta$ = interferon- $\beta$; IL-23 = interleukin-23; MCP-1 = monocyte chemotactic protein 1; TLR $3=$ toll-like receptor 3 ; NOD 2 = nucleotide binding oligomerization domain 2; TLR 2 = toll-like receptor 2 . Data are expressed as means \pm SD of triplicate samples. Statistical significance was determined by one-way ANOVA followed by Bonferroni's post-hoc test. ${ }^{*} P<0.05$

given alone (not shown). Combination of the three cytokines (stimulation with CCM) resulted in a two orders of magnitude higher mRNA expression that exceeded by far the sum of TNF- $\alpha$ and IL- $1 \beta$ signal intensities (Fig. 1B). A similar pattern was observed for the induction of NOS-2 and the release of nitrite from primary astrocytes (Falsig et al., 2004a, 2006). This indicates that IMA behaved similarly to murine astrocytes with respect to the cytokine response and, in particular, to the synergic activity of TNF- $\alpha$ and IL- $1 \beta$ with IFN- $\gamma$.

In a CCM stimulation time course, NOS-2 mRNA displayed a rapid rise after $4 \mathrm{~h}$, followed by a subsequent decline (Fig. 2A). Induction of mRNA coding for toll-like receptor-2 (TLR-2) was qualitatively similar to the situation observed with NOS-2. However, when compared quantitatively, the induction was far less pronounced compared with NOS-2 (Fig. 2B). In contrast to TLR-2, TLR-4 expression was not regulated by CCM stimulation (not shown).

For a more comprehensive comparison of the IMA subclone IMA 2.1 with primary mouse astrocytes, both cell populations were activated with CCM for $4 \mathrm{~h}$ and mRNA expression levels of a set of astrocyte-relevant marker genes were measured. The results indicated that the IMA 2.1 response resembles the response observed in primary astrocytes (Fig. 2C).

Following the analysis of a set of inflammation-related target genes on the mRNA level, we selected NOS-2 and COX-2 for a more detailed analysis on the protein and activity level. IMA 2.1 were stimulated with CCM for various time intervals. Both NOS-2 and COX-2 protein became detectable after $4 \mathrm{~h}$ of CCM 


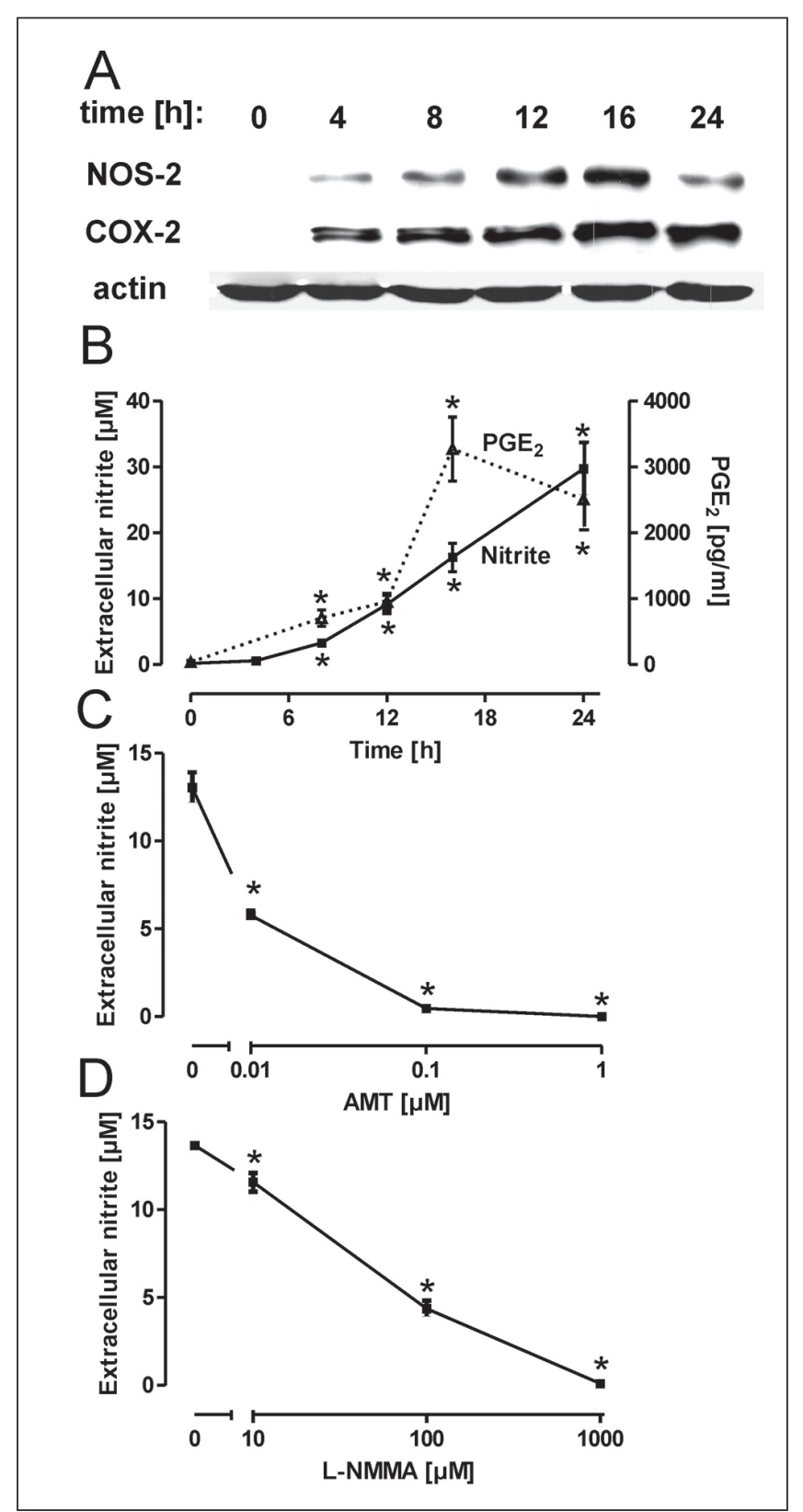

Fig. 3: Induction of cyclooxygenase-2 and nitric oxide synthase-2

IMA 2.1 were activated by CCM (IL-1 $\beta$, TNF-a, IFN- $\gamma$ ) for the time periods investigated. (A) The amount of cyclooxygenase-2 (COX-2) and nitric oxide synthase-2 (NOS-2) was evaluated on the protein level as shown by Western blot analysis. (B) Prostaglandin $\mathrm{E}_{2}\left(\mathrm{PGE}_{2}\right)$ as indicator for COX activity and nitrite as indicator for NOS activity were assessed in the respective cell culture supernatants. $(\mathrm{C}+\mathrm{D})$ To discriminate between NOS-2-dependent -NO formation and ${ }^{\bullet} \mathrm{NO}$ derived from constitutively expressed NOS proteins, the NOS-2 selective inhibitors AMT or L-NMMA were applied in different concentrations together with a cytokine mix for $16 \mathrm{~h}$. Nitrite served as indicator for NOS activity. Data are expressed as means $\pm S D$ of quadruplicate samples. Statistical significance was determined by one-way ANOVA followed by Bonferroni's post-hoc test. ${ }^{*} P<0.05$

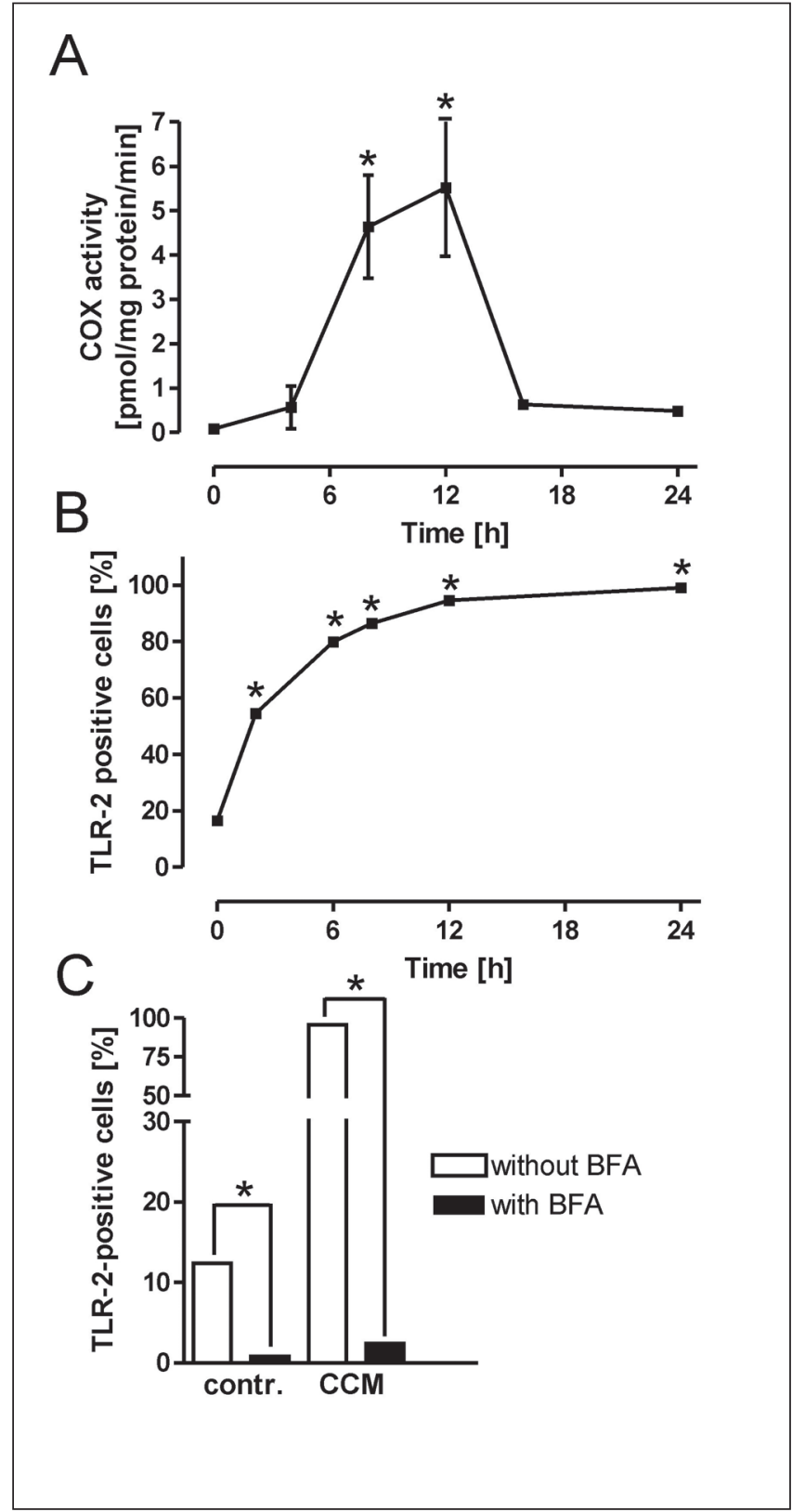

Fig. 4: Regulation of inflammation-relevant proteins

(A) IMA 2.1 were stimulated with CCM for the time intervals indicated, enzymatic activity of COX was directly assessed in cell lysates by the addition of ${ }^{14} \mathrm{C}$-labeled arachidonic acid.

(B) IMA 2.1 were activated with a cytokine mix as indicated, cells were stained with an anti-TLR-2 antibody and detected by flow cytometry.

(C) IMA 2.1 were treated with CCM, brefeldin A (BFA), or a combination of CCM and BFA for $16 \mathrm{~h}$, TLR-2 surface expression was analyzed by flow cytometry. Data are expressed as means \pm SD of quadruplicate samples. Statistical significance was determined by one-way ANOVA followed by Bonferroni's post-hoc test. ${ }^{*} P<0.05$ 
stimulation (Fig. 3A). Interestingly, NOS-2 mRNA peaked at $4 \mathrm{~h}$, while NOS-2 protein expression displayed a peak after 16 h. Similar observations have been made in other inflammatory cells, such as lipopolysaccharide-stimulated primary rat alveolar macrophages (Schildknecht, unpublished).

Accumulation of prostaglandin $\mathrm{E}_{2}\left(\mathrm{PGE}_{2}\right)$ in the supernatant served as an indirect indicator of COX activity. Unlike the gradual and continuing increase of nitrite as a marker of NOS activity, $\mathrm{PGE}_{2}$ demonstrated an increase up to $16 \mathrm{~h}$ after CCM stimulation. From then on, no further elevation was observed, despite a pronounced COX-2 protein expression (Fig. 3A,B). To test whether inducible NOS-2 was solely responsible for the nitrite accumulation, selective inhibitors of NOS-2, i.e., AMT and LNMMA, were applied in different concentrations together with $\mathrm{CCM}$ for $16 \mathrm{~h}$. The complete block of nitric oxide synthesis by these compounds suggests that no other NOS-isoform contributed to the observed $\bullet$ NO synthesis (Fig. 3C,D).

For a further investigation of the lack of $\mathrm{PGE}_{2}$ synthesis after $16 \mathrm{~h}$ of CCM activation, COX activity in homogenates of CCM-treated IMA was directly assessed by addition of the ${ }^{14} \mathrm{C}$-labeled substrate arachidonic acid, followed by separation and quantification of the prostanoids formed. In close correlation to the Western blot data in Fig. 3A, a constant increase in activity up to $12 \mathrm{~h}$ of CCM stimulation was observed, which was followed by a rapid decline in COX activity to almost baseline values (Fig. 4A). Similar observations were made in RAW 264.7 cells (Schildknecht et al., 2006) and primary rat alveolar macrophages (unpublished), in which a nitration and inactivation of COX-2 was observed. Although not investigated in detail in IMA 2.1 so far, it is likely that a similar mechanism is responsible for the observed effects, since we found that inactivation of COX-2 could be prevented with NOS-2 inhibitors (not shown).

We examined TLR-2 protein expression on the surface of CCM-stimulated IMA 2.1 by FACS analysis (Fig. 4B) in addition to the mRNA profiling (Fig. 2B). The surface expression continuously increased for up to $12 \mathrm{~h}$. The delay between the time course of mRNA and protein surface expression likely originated from a time period required for the synthesis and transport of newly formed TLR-2 from the Golgi- to the plasma-membrane. To test the importance of translocation, the secretory vesicle assembly blocker brefeldin A (BFA) was used. Under these conditions, a significant reduction in TLR-2 surface expression was detected. This inhibitory effect also was seen for the baseline TLR-2 surface expression of unstimulated cells (Fig. 4C). Together, these data indicate that IMA showed inflammatory responses similar to those of primary astrocytes or other inflammatory cells. Moreover, the data suggest the importance of activity measurements and protein quantification in addition to the mRNA profiling.

\subsection{Expression of immature astrocyte markers}

For an initial comparison of the marker expression and morphology of primary mouse astrocytes and IMA 2.1, cells were stained with antibodies selective for nestin and CD44 (Fig. 5A). These two proteins with cytoskeletal (nestin) and membraneous (CD44) localization allow the characterization of different morphological features. According to this, IMA 2.1 took the typical star-shape of astrocytes. Both markers were expressed in primary astrocytes and IMA 2.1, but the astrocytic extensions of IMA 2.1 were slightly shorter compared with primary astrocytes. Both, nestin and CD44, are not considered typical astrocyte markers (Kuegler et al., 2010, 2012), but are rather typical for immature precursors. However, they are often found in primary neonatal astrocyte cultures (which are commonly used for many experiments). The strong expression in IMA suggests their rather early-stage astrocyte precursor phenotype. For the assessment of general cell morphology, IMA 2.1 and primary astrocytes were stained with fluorescent phalloidin that selectively binds to F-actin. Confluent IMA 2.1 were additionally stained by calcein-AM $(1 \mu \mathrm{M} ; 30 \mathrm{~min})$ and the same region was photographed in the phase contrast mode (Fig. 5B). In order to test the functionality of the mature astrocyte marker glutamine synthase in IMA 2.1, cells were maintained under different culture conditions. In the presence of 2 $\mathrm{mM}$ glutamine, astrocytes had optimal growth conditions and they proliferated largely independent of the FCS concentrations in the culture. At lower glutamine concentrations, IMA increased their doubling time and became more dependent on high concentrations of FCS. Addition of glutamate compensated for the lack of glutamine supply, at least at high FCS concentrations. These data indicate that IMA were capable of enzymatic conversion of glutamate by glutamine synthase (Fig. 5C). Such a conversion of glutamate to glutamine is a typical function of astrocytes in the brain.

For further characterization of the enzymes involved in the glutamine/glutamate uptake and synthesis as well as other glial-related targets, mRNA expression was directly compared between primary mouse astrocytes, IMA 2.1 , and the astrocyteunrelated, murine control cell types $3 \mathrm{~T} 3$ or BV-2. In the selected set of markers, expression in primary astrocytes was in general much higher compared with IMA 2.1 (Fig. 6A,B). In most cases, the expression in IMA was similar to the one of a non-astrocytic cell (3T3-fibroblasts or BV-2 microglia) (Henn et al., 2009). The RNA data were confirmed also immunocytochemically, e.g., by an absence of staining for glial fibrillary acidic protein (GFAP). Four notable exceptions were found: aquaporin 4, a typical astrocyte marker was expressed 200-fold higher in IMA than in 3T3. Nestin was expressed in IMA to a similar extent as in primary astrocytes, which is consistent with immunostaining results. S100 $\beta$ and $\mathrm{Cnx} 43$, typical markers expressed early in astrogenesis, had an elevated expression relative to non-astrocytic markers. In summary, these data suggest that IMA resemble the GFAP-negative astrocytes that can be derived from stem cells (Kuegler et al., 2012) and are at a relatively immature stage.

\subsection{Metabolic capacity of IMA 2.1}

In addition to the descriptive phenotype markers, we also examined the actual metabolic competence of IMA. MAO-B activity was chosen because of its prime importance in neurotoxicology. IMA 2.1 were compared with HEK293, HeLa, the neuronal cell line LUHMES, and SH-SY5Y neuroblastoma cells with respect to MAO activity. Addition of the MAO-substrate kynuramine to 

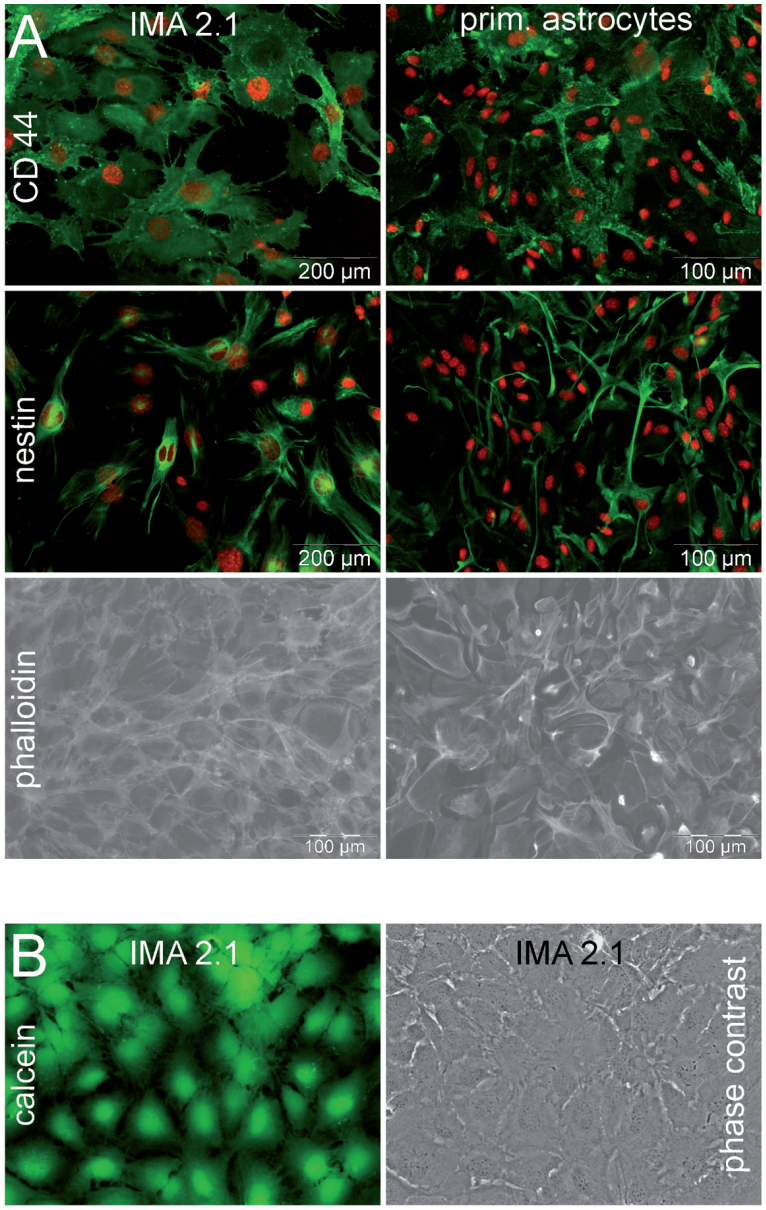

\section{C}

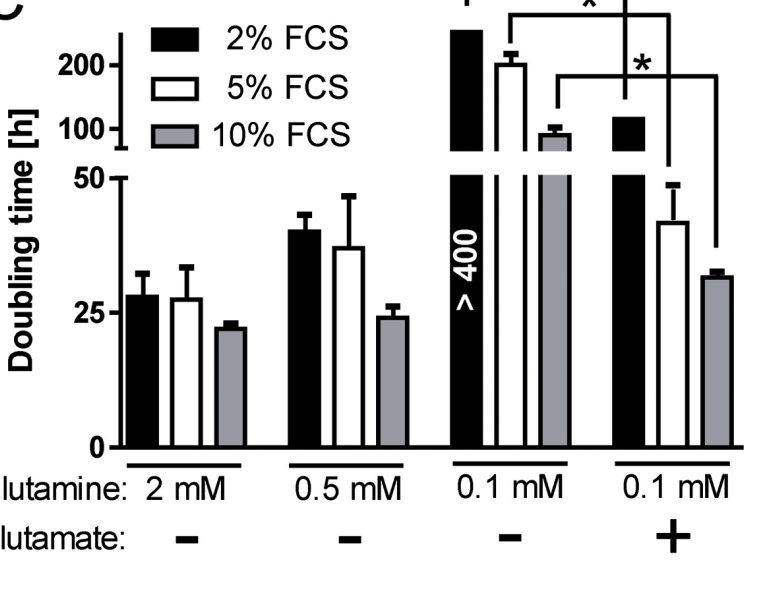

Fig. 5: Phenotypic and functional characteristics of IMA 2.1 (A) IMA 2.1 or primary murine astrocytes were fixed, permeabilized, and stained with antibodies against the cell surface glycoprotein CD44 and the intermediate filament protein nestin (green). Nuclei were labeled with $\mathrm{H}-33342$ (red). Alternatively, fluorescence-conjugated phalloidin, selectively binding to F-actin, was used for visualization of general cell morphology of IMA 2.1 and primary astrocytes.

(B) Additionally, living IMA 2.1 were labeled with calcein-AM ( $1 \mu \mathrm{M} ; 30 \mathrm{~min}$ ), the same region was also photographed in the phase contrast mode.

(C) IMA 2.1 were seeded at a density of 10,000 cells $/ \mathrm{cm}^{2}(2 \%$ FCS) and 5,000 cells $/ \mathrm{cm}^{2}(5 \%$ and $10 \%$ FCS) in the presence of different serum concentrations ( $2 \%, 5 \%, 10 \%)$. For each serum concentration, variable glutamine/glutamate levels were added, cells were allowed to grow for a total of 10 days and the number of cells was determined manually every $12 \mathrm{~h}$. The doubling time was calculated from the growth curves. Doubling time for cells grown with $2 \%$ serum and $0.1 \mathrm{mM}$ glutamine could not be determined as cells did not tolerate these growth conditions. The value was set to $270 \mathrm{~h}$ for graphical representation. Data are expressed as means $\pm S D$ of quadruplicate samples. Statistical significance was determined by one-way ANOVA followed by Bonferroni's post-hoc test. ${ }^{*} P<0.05$ 
homogenized cells indicated a significantly higher MAO activity in IMA compared with the other cell types investigated (Fig. 7A). Thus, as in the case of NOS-2 and TLR-2, the protein data did not correlate with the mRNA data. For a potential application of IMA 2.1 in co-culture models together with dopaminergic neurons in the field of Parkinson's disease research, conversion of the parkinsonian toxin MPTP into the dopaminergic neurotoxin $\mathrm{MPP}^{+}$(Fig. 7B) was investigated in IMA 2.1.

Addition of MPTP to IMA 2.1 led to a time-dependent conversion into its active metabolite $\mathrm{MPP}^{+}$. The reaction intermediate $\mathrm{MPDP}^{+}$was also detectable in the cell culture supernatant (Fig. 7C). The same experiment was then run with primary mouse astrocytes that displayed a similar MPTP conversion rate compared with IMA 2.1 (Fig. 7D). To identify the MAOisoform responsible for the observed MPTP conversion by IMA 2.1, cells were pretreated with the MAO-A-selective inhibitor moclobemide, the MAO-B-selective inhibitor (-)-deprenyl, or the isoform-unselective inhibitor pargyline for $30 \mathrm{~min}$ before MPTP was added. These experiments indicated an almost exclusive contribution of MAO-B to the observed conversion of MPTP (Fig. 7E). For a direct comparison of the influence of proinflammatory conditions on MAO-B activity, IMA 2.1 were pre-treated with CCM for $6 \mathrm{~h}$ for full activation. When MPTP was added as MAO-B substrate, $\mathrm{MPP}^{+}$was detected as readout in the supernatant and revealed no significant difference between the control and the CCM group (Fig. 7F). These findings correlate well with a large body of literature, examining MPTP in the in vivo mouse model.

\subsection{Compatibility with neuronal co-cultures}

Finally, we tested whether IMA 2.1 can be used in a co-culture model together with the human dopaminergic neuronal cell line LUHMES. For direct comparison, primary astrocytes or IMA 2.1 were grown until confluency was reached. Then, predifferentiated LUHMES were added and differentiated for four days. The morphology of LUHMES was analyzed by staining of the neuronal marker protein $\beta$-III-tubulin. As illustrated in Figure 8, differentiated LUHMES grown alone, or on top of either primary astrocytes or IMA 2.1 displayed similar neurite structures and cell morphology. These observations indicate that IMA 2.1 can be applied in co-culture models either in direct contact with neurons as illustrated herein, or spatially separated in a transwell co-culture system.

\section{Discussion}

In an aging population, the incidence of chronic neurodegenerative diseases such as PD or AD is constantly increasing. This results in intensified basic and clinical research in this field, as evidenced by the higher use of experimental animals for basic research, and in particular, the widespread generation and use of new transgenic animal models (Leist et al., 2008). In the present manuscript, we characterized the mouse cell line IMA with respect to its potential as substitute for primary astrocyte cultures. Particular emphasis was laid on IMA's response to proinflammatory stimuli and its ability to serve as model for the

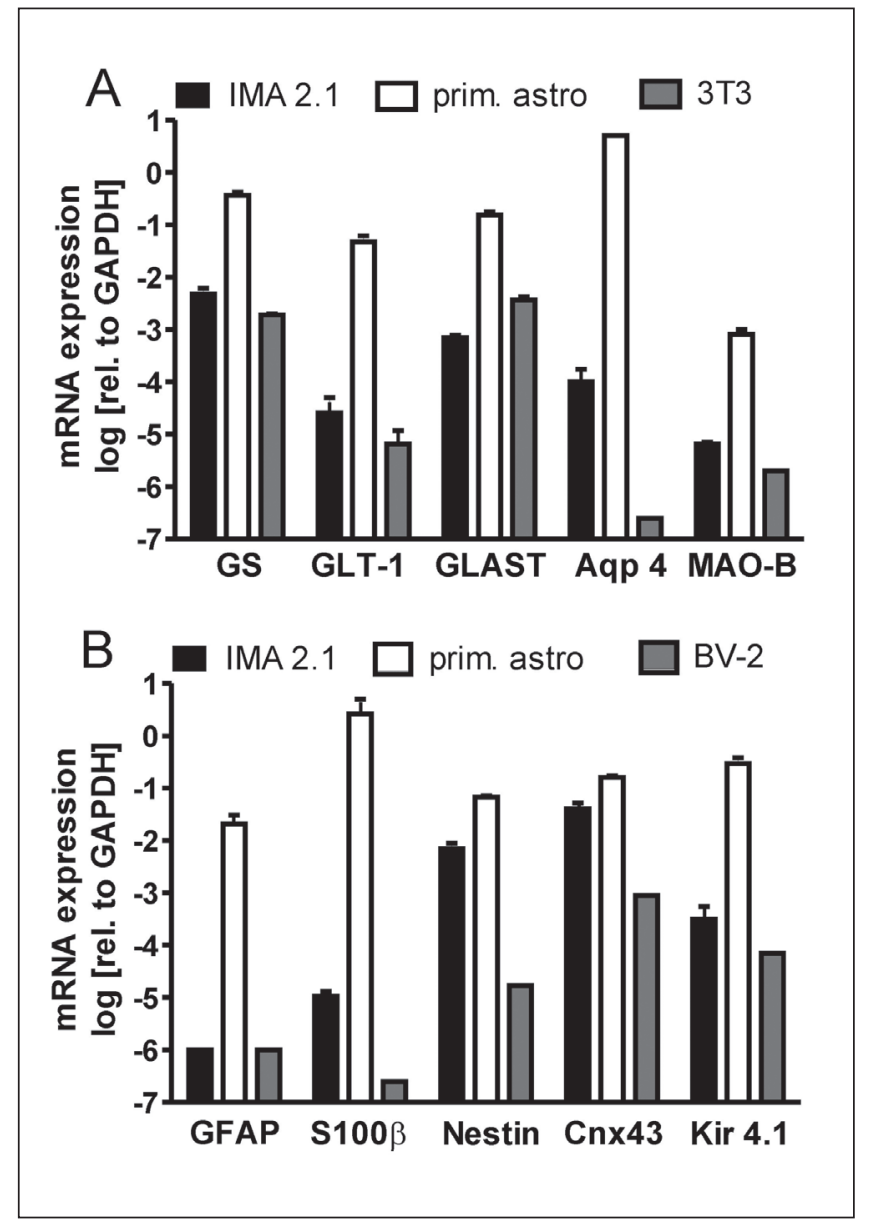

Fig. 6: Comparison of mRNA expression in IMA 2.1, primary mouse astrocytes, and non-astrocytic cells Total mRNA was extracted as template for reverse transcription and PCR amplification. Data are shown relative to GAPDH expression in the same well. In (A), ЗТ3 cells served as non-astrocytic reference, in (B) BV-2 was used as reference. GS= glutamine synthase; GLT-1 = glutamate transporter 1 ; GLAST = glutamate-aspartate transporter; Aqp $4=$ aquaporin 4; MAO-B = monoamine oxidase $\mathrm{B}$; GFAP = glial fibrillary acidic protein; $\mathrm{Cnx43}=$ connexin 43; Kir 4.1 = inwardly rectifying $\mathrm{K}^{+}$channel. Data are means \pm SEM of three different cultures.

conversion of the parkinsonian toxin MPTP. In the field of PD research, in vitro models that recapitulate these two events are desired.

The standard method to obtain astrocytes is the establishment of primary cell cultures by isolation of cells from brain tissue of newborn animals. This costly and time-consuming procedure represents a clear logistic bottleneck in molecular mechanistic research on astrocyte function. Hence, replacement of primary astrocytes by an adequate cell line has a high potential to advance our knowledge about astrocyte biology and, at the same time, contributes to reduce the number of experimental animals used. For successful replacement, new in vitro models however 


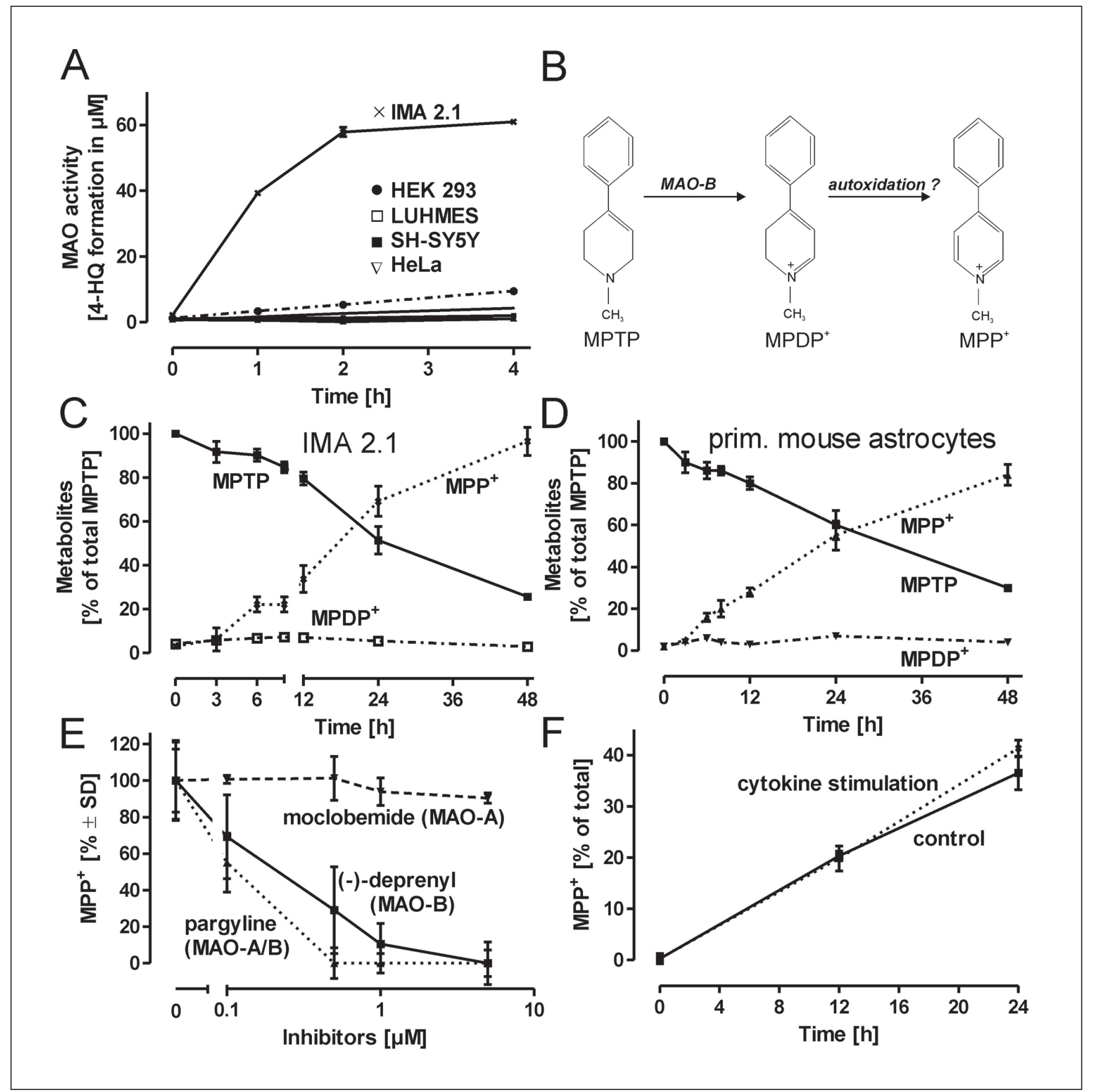

Fig. 7: Monoamine oxidase activity and MPTP metabolism

(A) For a direct comparison of monoamine oxidase (MAO) activity, homogenates (1 mg/ml protein) of IMA 2.1, the neuronal cell lines LUHMES and SH-SY5Y, or HeLa and HEK 293 were incubated with the MAO substrate kynuramine (100 $\mu \mathrm{M})$ for $2 \mathrm{~h}$. The reaction product hydroxychinoline was measured fluorimetrically and the resulting concentrations were plotted. (B) Overview on the structures of 1-methyl-4-phenyl-1,2,3,6-tetrahydropyridine (MPTP), the reaction intermediate 1-methyl-4-phenyl-2,3-dihydropyridinium (MPDP+) and the active toxin 1-methyl-4-phenylpyridinium $\left(\mathrm{MPP}^{+}\right)$. $(\mathrm{C}+\mathrm{D}) \mathrm{MPTP}(20 \mu \mathrm{M})$ was added to IMA 2.1 or to primary mouse astrocyte cultures. At the time points indicated, supernatant was collected and MPTP, MPDP ${ }^{+}$, and MPP ${ }^{+}$were measured by HPLC. (E) To investigate which MAO isoform accounts for the observed activity, MPTP $(20 \mu \mathrm{M})$ was added together with the MAO-A selective inhibitor moclobemide, the MAO-B selective inhibitor (-)-deprenyl, or the isoform-unselective inhibitor pargyline. After $24 \mathrm{~h}, \mathrm{MPP}+$ was evaluated in the supernatant by HPLC. (F) For a comparison on the impact of inflammation on MAO-B activity, IMA were stimulated with CCM for $6 \mathrm{~h}$. Then, MPTP $(20 \mu \mathrm{M})$ was added, and MPP+ was detected by HPLC in the supernatant after the time periods indicated. Data are expressed as means $\pm S D$ of quadruplicate samples. 


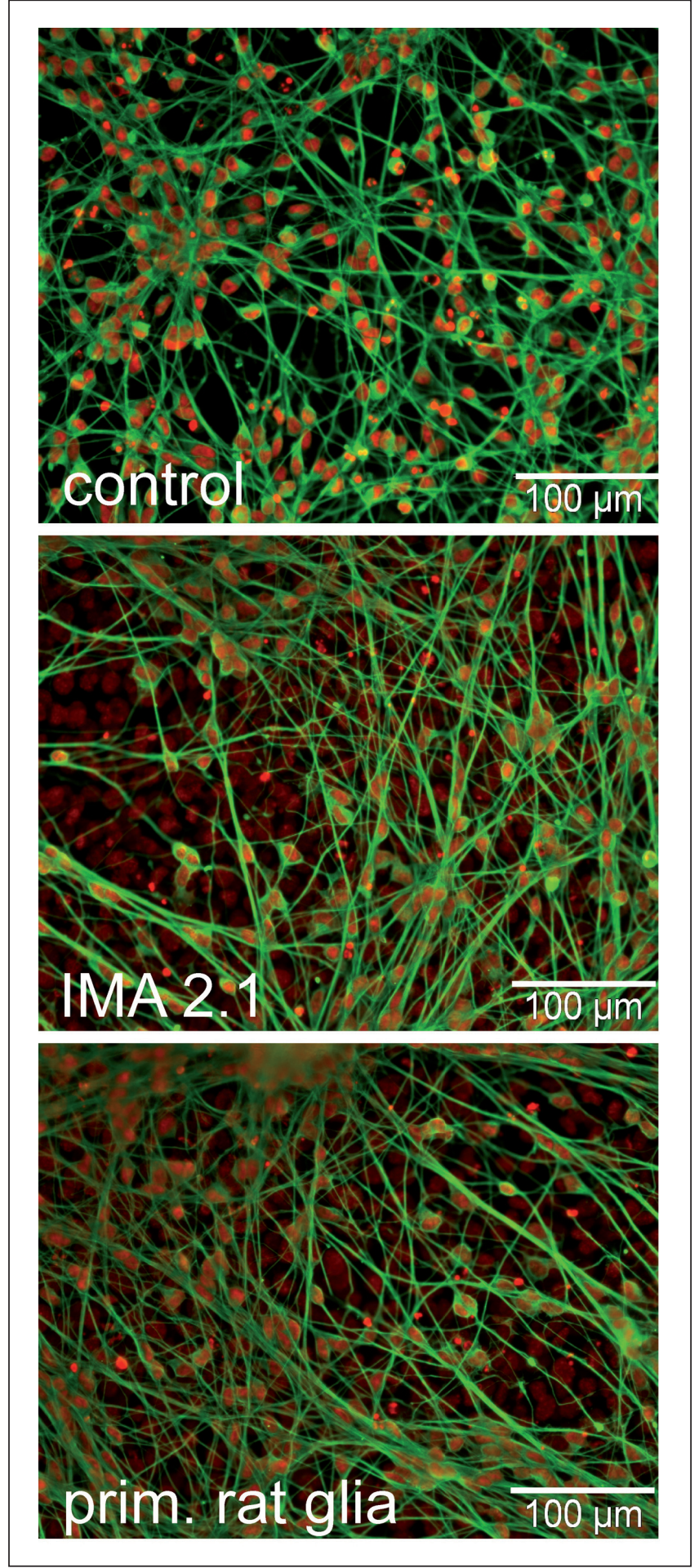

Fig. 8: Application of IMA 2.1 in co-culture models

Neuronal LUHMES cells were either differentiated alone (control), or on top of confluent layers of IMA 2.1 or primary rat glia cells. For a phenotypic assessment, LUHMES were stained for neuronspecific $\beta$-III-tubulin (green), nuclei were stained with Hoechst $\mathrm{H}-33342$ (red). require acceptance by the scientific community. Previous astrocyte cell lines failed to become widely distributed, and they failed to be adopted by the research community.

Primary cultures of astrocytes are undoubtedly closer than any immortalized cell line to an in vivo situation, however also primary cells have, aside from limited availability, clear cut limitations. For example, contamination with microglia can significantly affect the outcome of an investigation (Saura, 2007). It is also important to realize that brain region-dependent differences in astrocytic phenotype exist that can have a profound impact on the observations made. Moreover, it has been clearly demonstrated that the commonly used cultures of neonatal astrocytes differ vastly from adult astrocytes in the brain (Zhang and Barres, 2010).

In the present study, we evaluated the mouse astrocyte cell line IMA with respect to its applicability in inflammation studies and for its suitability as an MPTP conversion model. In comparison to primary astrocytes, IMA are easy to handle, do not require laborious isolation methods that usually lead to batch-to-batch variations, and are stable in their phenotype over years, which is a prerequisite for the reproducibility of results and inter-laboratory comparability. This aspect also opens the field for potential applications in validation assays. We also observed that, in contrast to other cell types, genetic overexpression by transfection or electroporation, or the siRNA-mediated knockdown of targets of interest can be performed in IMA (data not shown).

The suitability of IMA as inflammation model was assessed by the detection of NF- $\kappa B$ translocation into the nucleus, which represents one of the key events involved in the induction of so-called immediate early genes. The detection method by an automated microscope system on the single cell level showed that all IMA reacted simultaneously to different cytokines. Such a homogeneous cell population is of great advantage for many biochemical and signal transduction studies. For a general overview of the inflammatory competence, we have chosen changes of mRNA levels as readout, as suggested previously (Kuegler et al., 2010). However, characterization based on transcript levels alone represented only a first overview. Further information on the actual expression, localization, and activity of a protein or enzyme was therefore presented here for NOS-2, MAO-B, or TLR-2, as examples.

The low GFAP mRNA expression, and the absence of GFAP staining (not shown) in IMA might raise concerns about the astrocytic origin of the cells. However, the original cell pool stained uniformly positive for GFAP directly after transduction. During the four passages thereafter, the expression of GFAP was lost, but the cells maintained the same morphologic features. It has previously been reported that GFAP expression depends highly on the proliferation status of cells. Accordingly, GFAP was reported to be downregulated in a proliferating astrocyte cell line (Price et al., 1999). This might explain the low abundance observed in IMA. It is also becoming increasingly clear that a large subpopulation of astrocytes does not express GFAP, also under in vivo conditions. Confluent cells kept for more than a week displayed elevated levels of GFAP that were however still significantly lower compared with the expression in pri- 
mary astrocytes. The established cell line IMA 2.1 obviously also lacks some genuine astrocyte functions such as functional expression of glutamine synthase, as well as specific uptake of glutamate. These observations suggest limitations of IMA 2.1 in the field of glutamate/EAAT-toxicity research.

For potential applications in parkinsonian MPTP models, we compared the MAO-dependent conversion of MPTP by IMA and primary mouse glial cells that indicated a comparable MAO-B activity in IMA when adjusted to equal total protein content. The IMA cell line therefore represents an easily accessible platform for mechanistic studies on MPTP conversion.

In order to closer simulate the situation in the brain, we then tested whether IMA can form co-cultures with the human dopaminergic cell line LUHMES. The LUHMES cell line that can be differentiated into post-mitotic neurons with a dopaminergic phenotype is very sensitive to culture conditions. Therefore, the successful co-cultivation was initially surprising, and these data indicate that IMA 2.1 can most likely also be used in combination with other neuronal cell types (Stiegler et al., 2011; Zimmer et al., 2011). The rationale for using a human neuronal cell line is based on the intended use of such co-cultures for human disease modeling. The relevant target cells for most diseases are neurons. To our current knowledge, LUHMES cells represent one of the most favorable in vitro models of dopaminergic neurons. We recently characterized this cell line (Scholz et al., 2011; Schildknecht et al., 2009) extensively, and we believe that no mouse cell line with similar features is available at present. Vice versa, the use of human astrocytes is much less established than the one of murine astrocytes, and the cells are less accessible. Notably, the combination of cells from different species does not result in histoincompatibility reactions, as long as no $\mathrm{T}$ cells are present. Moreover, most metabolites that are exchanged, in addition to most neurotransmitters, lipid mediators, growth factors and xenobiotic metabolites relevant to the co-culture, are not species-specific. Therefore, combining cell types of different species created no difficulties. We are aware of the disadvantages of the two-species system for the study of certain immune mediators (e.g., IFN- $\gamma$ ) that are species-specific. On the other hand, two species systems also have enormous advantages, as certain cytokines derived from one of the cell types may be blocked selectively, without influencing the same cytokine from the other cell type (e.g., human or murine CCL-5), and especially the regulation of transcripts can be followed for each cell type selectively by choosing appropriate PCR primers. Such advantages can be very important for mechanistic studies of reciprocal cell-type interactions. In preliminary results (not shown), we observed that MPTP addition to the co-culture model of IMA and LUHMES leads to a time-dependent conversion into the active toxin $\mathrm{MPP}^{+}$that subsequently is taken up by LUHMES cells and leads to a selective neurite degeneration and ultimately cell death while viability of IMA, in which the conversion takes place, is not affected. These findings however require further investigations and confirmation.

The suitability of IMA for co-culture models in combination with neurons opens a promising perspective for a new in vitro system to study cellular interactions that may be relevant for disease. For instance, mechanistic and kinetic aspects of the toxicity of the Parkinsonism-inducing toxicant MPTP may be studied, which is not possible in pure neuronal cultures.

\section{References}

Beckman, J. S. and Koppenol, W. H. (1996). Nitric oxide, superoxide, and peroxynitrite: the good, the bad, and ugly. Am J Physiol 271, C1424-1437.

Brown, G. C. and Neher J. J. (2010). Inflammatory neurodegeneration and mechanisms of microglial killing of neurons. Mol Neurobiol 41, 242-247.

Chang, P. L., Gunby, J. L., Tomkins, D. J., et al. (1986). Transformation of human cultured fibroblasts with plasmids carrying dominant selection markers and immortalizing potential. Exp Cell Res 167, 407-416.

Christiansen, S. H., Selige, J., Dunkern, T., et al. (2011). Combined anti-inflammatory effects of $\beta 2$-adrenergic agonists and PDE4 inhibitors on astrocytes by upregulation of intracellular cAMP. Neurochem Int 59, 837-846.

Di Monte, D. A., Wu, E. Y., Irwin, I., et al. (1991). Biotransformation of 1-methyl-4-phenyl-1,2,3,6-tetrahydropyridine in primary cultures of mouse astrocytes. $J$ Pharmacol Exp Ther 258, 594-600.

Falsig, J., Latta, M., and Leist, M. (2004a). Defined inflammatory states in astrocyte cultures: correlation with susceptibility towards CD95-driven apoptosis. J Neurochem 88, 181-193.

Falsig, J., Pörzgen, P., Lotharius, J., and Leist, M. (2004b). Specific modulation of astrocyte inflammation by inhibition of mixed lineage kinases with CEP-1347. J Immunol 173, 27622770 .

Falsig, J., Pörzgen, P., Lund, S., et al . (2006). The inflammatory transcriptome of reactive murine astrocytes and implications for their innate immune function. J Neurochem 96, 893-907.

Falsig, J., van Beek, J., Hermann, C., and Leist, M. (2008). Molecular basis for detection of invading pathogens in the brain. J Neurosci Res 86, 1434-1447.

Frisa, P. S., Goodman, M. N., Smith, G. M., et al. (1994). Immortalization of immature and mature mouse astrocytes with SV40 T antigen. $J$ Neurosci Re 39, 47-56.

Hansson, E. (1986). Primary astroglial cultures. A biochemical and functional evaluation. Neurochem Res 11, 759-767.

Henn, A., Lund, S., Hedtjärn, M., et al. (2009). The suitability of $\mathrm{BV} 2$ cells as alternative model system for primary microglia cultures or for animal experiments examining brain inflammation. ALTEX 26, 83-94.

Henn, A., Kirner, S., and Leist, M. (2011). TLR2 hypersensitivity of astrocytes as functional consequence of previous inflammatory episodes. J Immunol 186, 3237-3247.

Kettenmann, H. and Ransom, B. R. (ed.) (2005). Neuroglia. $2^{\text {nd }}$ edition. New York, USA: Oxford University Press.

Kim, H. J. and Magrané, J. (2011) Isolation and culture of neurons and astrocytes from the mouse brain cortex. Methods Mol Biol 793, 63-75.

Kuegler, P. B., Zimmer, B., Waldmann, T., et al. (2010). Markers of murine embryonic and neural stem cells, neurons and 
astrocytes: reference points for developmental neurotoxicity testing. ALTEX 27, 17-42.

Kuegler,P. B., Baumann, B.A.,Zimmer, B., et al .(2012). GFAPindependent inflammatory competence and trophic functions of astrocytes generated from murine embryonic stem cells. Glia 60, 218-228.

Langston, J. W., Irwin, I., Langston, E. B., and Forno, L. S. (1984). Pargyline prevents MPTP-induced parkinsonism in primates. Science 225, 1480-1482.

Lee, S. C., Liu, W., Dickson D. W., et al. (1993). Cytokine production by human fetal microglia and astrocytes. Differential induction by lipopolysaccharide and IL-1 beta. Immunol 150, 2659-2667.

Leist, M., Kadereit, S., and Schildknecht, S. (2008). Food for thought ... on the real success of $3 \mathrm{R}$ approaches. ALTEX 25, 17-32.

Leist, M., Efremova, L., and Karreman, C. (2010). Food for thought ... considerations and guidelines for basic test method descriptions in toxicology. ALTEX 27, 309-317.

Lotharius, J., Falsig, J., van Beek, J., et al. (2005). Progressive degeneration of human mesencephalic neuron-derived cells triggered by dopamine-dependent oxidative stress is dependent on the mixed-lineage kinase pathway. J Neurosci 25, 6329-6342.

Lund, S., Porzgen, P., Mortensen, A. L., et al. (2005). Inhibition of microglial inflammation by the MLK inhibitor CEP-1347. J Neurochem 92, 1439-1451.

Lund, S., Christensen, K. V., Hedtjärn, M., et al. (2006). The dynamics of the LPS triggered inflammatory response of murine microglia under different culture and in vivo conditions. $J$ Neuroimmunol 180, 71-87.

Meeuwsen, S., Persoon-Deen, C., Bsibsi, M., et al. (2003). Cytokine, chemokine and growth factor gene profiling of cultured human astrocytes after exposure to proinflammatory stimuli. Glia 43, 243-253.

Murphy, S., Pearce, B., Jeremy, J., and Dandona, P. (1988). Astrocytes as eicosanoid-producing cells. Glia 1, 241-245.

Pöltl, D., Schildknecht, S., Karreman, C., and Leist, M. (2011). Uncoupling of ATP-depletion and cell death in human dopaminergic neurons. Neurotoxicology. Dec 19, Epub ahead of print.

Price, T. N., Burke, J. F., and Mayne, L. V. (1999). A novel human astrocyte cell line (A735) with astrocyte-specific neurotransmitter function. In Vitro Cell Dev Biol Anim 35, 279288.

Ransom, B. R., Kunis, D. M., Irwin, I., and Langston J. W. (1987). Astrocytes convert the parkinsonism inducing neurotoxin, MPTP, to its active metabolite, $\mathrm{MPP}^{+}$. Neurosci Lett 75, 323-328

Roqué, P. J., Guizzetti, M., Giordano, G., and Costa, L. G. (2011). Quantification of synaptic structure formation in cocultures of astrocytes and hippocampal neurons. Methods Mol Biol 758, 361-390.

Ryder, E. F., Snyder, E. Y., and Cepko, C. L. (1990). Establish- ment and characterization of multipotent neural cell lines using retrovirus vector-mediated oncogene transfer. J Neurobiol $21,356-375$.

Saura, J. (2007). Microglia cells in astroglia cultures: a cautionary note. J. Neuroinflamm 4, 26.

Schildknecht, S., Heinz, K., Daiber, A., et al. (2006). Autocatalytic tyrosine nitration of prostaglandin endoperoxide synthase-2 in LPS-stimulated RAW 264.7 macrophages. Biochem Biophys Res Commun 340, 318-325.

Schildknecht, S., van der Loo, B., Weber, K., et al. (2008). Endogenous peroxynitrite modulates PGHS-1-dependent thromboxane A2 formation and aggregation in human platelets. Free Radic Biol Med 45, 512-520.

Schildknecht, S., Pöltl, D., Nagel, D. M., et al. (2009). Requirement of a dopaminergic neuronal phenotype for toxicity of low concentrations of 1-methyl-4-phenylpyridinium to human cells. Toxicol Appl Pharmacol 241, 23-35.

Schildknecht, S., Pape, R., Müller, N., et al. (2011). Neuroprotection by minocycline caused by direct and specific scavenging of peroxynitrite. J Biol Chem 286, 4991-5002.

Scholz, D., Pöltl, D., Genewsky, A., et al. (2011). Rapid, complete and large-scale generation of post-mitotic neurons from the human LUHMES cell line. J Neurochem 119, 957-971.

Stiegler, N. V., Krug, A. K., Matt, F., and Leist, M. (2011). Assessment of chemical-induced impairment of human neurite outgrowth by multiparametric live cell imaging in high-density cultures. Toxicol Sci 121,73-87.

Zhang, Y. and Barres, B. A. (2010). Astrocyte heterogeneity: an underappreciated topic in neurobiology. Curr Opin Neurobiol 20, 588-594.

Zimmer, B., Schildknecht, S., Kuegler, P. B. et al. (2011). Sensitivity of dopaminergic neuron differentiation from stem cells to chronic low-dose methylmercury exposure. Toxicol Sci $121,357-367$

\section{Acknowledgments}

We would like to thank Prof. Dr Klaus Pfizenmaier (University of Stuttgart) for his fruitful discussion during the preparation of the manuscript. The work was facilitated by grants from the Doerenkamp-Zbinden foundation, the Federal State of BadenWürttemberg and the DFG. SFB969, RTG1331, and KoRS-CB are acknowledged for their support.

\section{Correspondence to}

Stefan Schildknecht, PhD

University of Konstanz

PO Box M657

78457 Konstanz

Germany

Phone: +49 7531885053

Fax: +497531885039

e-mail: Stefan.Schildknecht@uni-konstanz.de 